Louisiana State University

LSU Digital Commons

Faculty Publications

Department of Physics \& Astronomy

6-1-2016

\title{
A recoil separator for nuclear astrophysics SECAR
}

\author{
G. P.A. Berg \\ University of Notre Dame \\ D. W. Bardayan \\ University of Notre Dame \\ J. C. Blackmon \\ Louisiana State University \\ K. A. Chipps \\ ORNL Physics Division \\ M. Couder \\ University of Notre Dame
}

See next page for additional authors

Follow this and additional works at: https://digitalcommons.Isu.edu/physics_astronomy_pubs

\section{Recommended Citation}

Berg, G., Bardayan, D., Blackmon, J., Chipps, K., Couder, M., Greife, U., Hager, U., Montes, F., Rehm, K., Schatz, H., Smith, M., Wiescher, M., Wrede, C., \& Zeller, A. (2016). A recoil separator for nuclear astrophysics SECAR. Nuclear Instruments and Methods in Physics Research, Section B: Beam Interactions with Materials and Atoms, 376, 165-167. https://doi.org/10.1016/j.nimb.2016.02.009

This Article is brought to you for free and open access by the Department of Physics \& Astronomy at LSU Digital Commons. It has been accepted for inclusion in Faculty Publications by an authorized administrator of LSU Digital Commons. For more information, please contact ir@lsu.edu. 


\section{Authors}

G. P.A. Berg, D. W. Bardayan, J. C. Blackmon, K. A. Chipps, M. Couder, U. Greife, U. Hager, F. Montes, K. E. Rehm, H. Schatz, M. S. Smith, M. Wiescher, C. Wrede, and A. Zeller 


\title{
A recoil separator for nuclear astrophysics SECAR
}

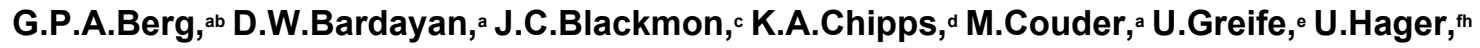 \\ F.Montes $f$ K.E.Rehm, ${ }^{9}$ H.Schatz, ${ }^{\text {th }}$ M.S.Smith, ${ }^{d}$ M.Wiescher, ${ }^{a}$ C.Wrede, ${ }^{f}$ A.Zeller ${ }^{f}$ \\ a Dept. of Physics, University of Notre Dame, Notre Dame, IN 46556, USA \\ ${ }^{b}$ The Joint Inst. for Nucl. Astrophysics, Univ. of Notre Dame, Notre Dame, IN 46556, USA \\ c Dept. of Physics and Astronomy, Louisiana State University, Baton Rouge, LA 70803, USA \\ a Physics Division, Oak Ridge National Laboratory, Oak Ridge, TN 37831, USA \\ e Dept. of Physics, Colorado School of Mines, Golden, CO 80401, USA \\ ${ }^{f}$ National Superconducting Cyclotron Laboratory, Michigan State University, East Lansing, MI 48824, \\ USA \\ g Physics Division, Argonne National Laboratory, Argonne, IL 60439, USA \\ ${ }^{h}$ Dept. of Physics and Astronomy, Michigan State University, East Lansing, MI 48824, USA
}

\begin{abstract}
A recoil separator SECAR has been designed to study radiative capture reactions relevant for the astrophysical rp-process in inverse kinematics for the Facility for Rare Isotope Beams (FRIB). We describe the design, layout, and ion optics of the recoil separator and present the status of the project.

\section{Keywords}

Recoils mass separator; Wien filter; Radiative , $\alpha$, and $p$ capture. 


\section{Introduction}

The explosive nuclear burning of hydrogen at high temperatures and densities on the surface of accreting white dwarfs and neutron stars gives rise to a number of observable nuclear explosions including Novae [1] or X-ray bursts [2]. Recent astronomical observations provide unprecedented information, for example, on atomic abundances in Nova ejecta and time structure in X-ray bursts. Interpretation of these data requires an understanding of the nuclear processes during the explosive events and, therefore, information on the reactions of unstable, proton-rich nuclei with hydrogen and helium.

We developed SECAR (SEparator for CApture Reactions), a recoil separator with the sensitivity needed to measure very low $(p, \gamma)$ and $(\alpha, \gamma)$ rp-process reaction rates directly at astrophysical energies in inverse kinematics for target mass from $A=15$ to $A=65$. This requires a large angle acceptance of \pm 25 mrad given by the ${ }^{15} \mathrm{O}(\alpha, \gamma){ }^{19} \mathrm{Ne}$ reaction at $0.5 \mathrm{MeV}$ center of mass energy to accommodate the kinematic angle cone and the angle spread due to multiple scattering in the windowless gas target, so that all recoils are transmitted. The very high mass resolving power $\mathrm{m} / \mathrm{dm} \geqslant 750$ is given by the ${ }^{65} \mathrm{As}(\mathrm{p}, \mathrm{y})^{66} \mathrm{Se}$ reaction and is more than a factor of 2 larger compared to existing recoil separators to achieve a beam rejection of about $10^{-13}$. An additional rejection of about $10^{-4}$ is provided by the detector system.

SECAR is designed to be a next-generation device, with specifications to match the beam production capabilities of FRIB and the scientific needs of the community. These specifications will significantly exceed the two major separators in the field, the Detectors of Recoils And Gammas of Nuclear reactions (DRAGON)[4] at TRIUMF ISAC and the Daresbury Recoil Separator (DRS)[5] at ORNL. The viability of measuring proton capture reactions in inverse kinematics with a recoil separator was demonstrated in 1991 with a small system at Caltech[6]. In addition to DRAGON and DRS other separators have been built for this technique, including ARES [7], ERNA [8], and St. George [9]. SECAR will initially operate at the ReA3 rare isotope (RI) beam facility at NSCL, MSU, taking advantage of its unique capability to produce a wide range of radioactive beams. SECAR will achieve its full potential with the intense radioactive beams that can be produced at the Facility for Rare Isotope Beams (FRIB), a next generation facility currently under construction at Michigan State University.

\section{Design of the recoils separator}

The floor plan of the recoil separator SECAR is shown in Fig. 1. The system consists of four 1 Introduction, 2 Design of the recoils separator, 3 lon optics, 4 Project status with four focal planes (FP1- FP4) at the end of each section. Section 1 starts at the windowless gas jet target JENSA [3] and ends at focal plane F1. JENSA is already constructed and installed and is presently used as stand alone facility. Only minor modifications are needed to adapt JENSA for the use with SECAR. Sufficiently high dispersion at FP1 is provided to select a single charge state of the beam and reaction products or recoils. Section 2 with velocity filter WF1 has a mass resolving power of about $\mathrm{m} / \mathrm{dm}=750$ that is reduced to about $\mathrm{m} / \mathrm{dm}=500$ by higher order aberrations. To achieve this there is an achromatic focus at FP2 where the beam and recoils are separated. Most of the beam is stopped while the recoils, still riding on a tail of the beam, 
will be fully transmitted through Section 3 . This section includes a second velocity filter increasing the mass resolving power to the required $\mathrm{m} / \mathrm{dm}=750$. In the focal plane $\mathrm{F} 3$ the tail of the beam is further separated from the recoils and stopped by a slit. The final Section 4 has two functions (a) to reduce background and (b) to prepare the beam for full transmission through the detector system. The background consists of beam particles scattered from upstream beam apertures, scattering and charge exchange from the residual gas, and beam tails. The complete system consists of 2 velocity filters, 8 dipole, 14 quadrupole, 3 hexapole, and 1 octupole magnets. In addition there is a combined function magnet (Q1 + Hex) that includes a quadrupole and hexapole at the very beginning of the system.

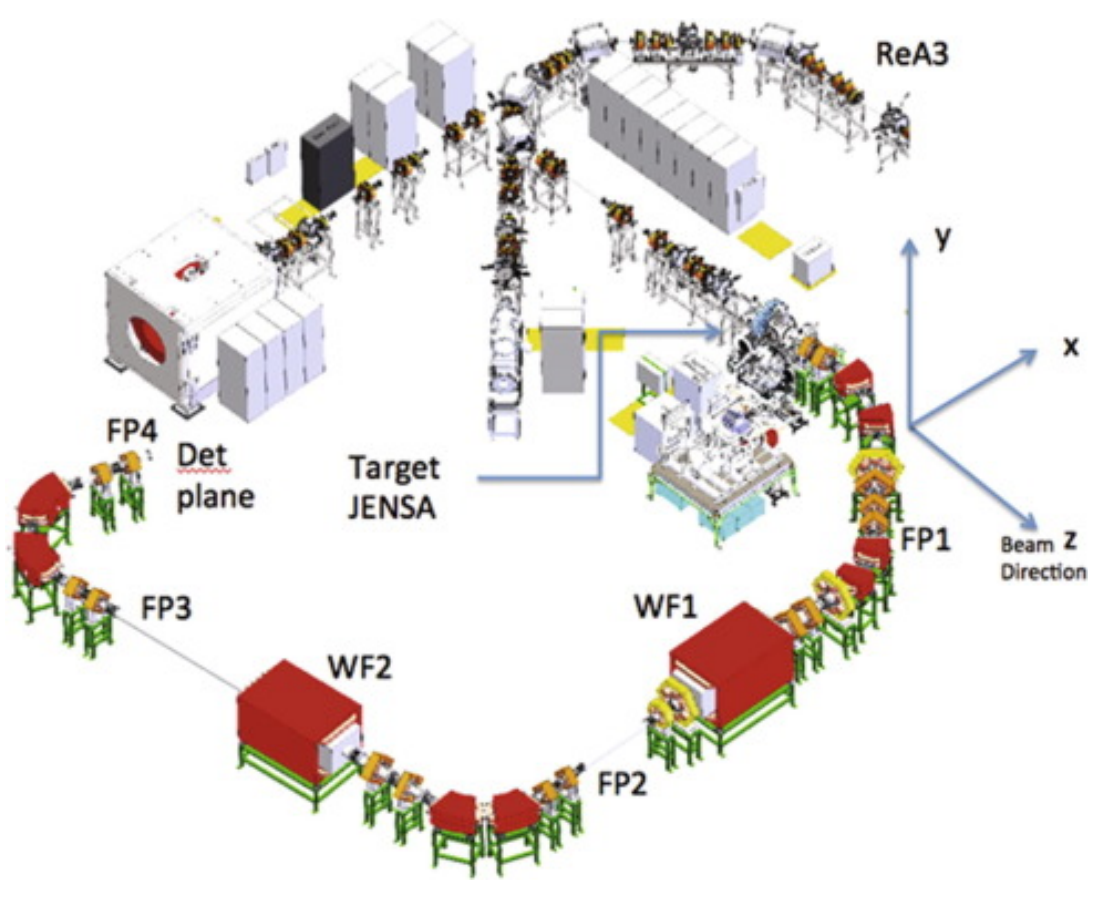

Fig. 1. SECAR layout in the ReA3 Hall at MSU. The beam enters the hall from the top right, impinges on the JENSA gas jet target. The recoils and unreacted projectiles enter the first section that selects a single charge state. Two additional sections each have a velocity filter (WF1, WF2) for projectile rejection. The

final "cleanup" section features two dipoles and a drift section before the recoils enter the focal plane detection system.

\section{Ion optics}

One of the critical design parameters of SECAR is the high mass resolving power.The ionoptical concept was developed and optimized using the code COSY Infinity [10]. The first-order mass resolving power is defined by $R_{m}=R_{17} /\left(R_{11}{ }^{*} 2 x_{0}\right)$ where $R_{17}$ is the mass dispersion, $R_{11}$ the magnification and $2 \mathrm{x}_{0}$ the full object spot size. However, a mass resolving power as high as $R_{m}$ is only achieved if the higher order $(\mathrm{HO})$ aberrations are corrected to negligible contributions. Ideally, the minimization of the image spot size including $\mathrm{HO}$ aberrations would be performed using a large number of rays with realistic image phase space distribution. Since this is not practical mainly because of the long computing time, we defined an array of 189 
"characteristic rays" to derive the image size, as a compromise. These rays cover the maximum design acceptances in an equal-distance grid-type distribution of the horizontal $( \pm 25 \mathrm{mrad})$, vertical $( \pm 25 \mathrm{mrad})$, and energy acceptances $( \pm 3.1 \%)$ as well as horizontal and vertical target spot sizes of $\pm 0.75 \mathrm{~mm}$. The image size is defined as the maximum of distances between all 189 rays in the horizontal plane. A comparison of the image sizes of the optimized ion-optics using this method and Monte Carlo calculations with $10^{4}$ rays with Gaussian phase-space distributions showed reasonable agreement. The ion-optical concept of SECAR was optimized sequentially for orders 1, 2, 3 and 4. This is accomplished by using appropriate shapes of the dipole magnet entrances and exit edges. In addition 3 hexapole and 1 octupole magnets are included in Section 2 for further improvement and flexibility. The inclusion of orders 5 and 6 do not change or improve the ion-optics significantly, notably the mass resolving power. The results of the final, optimized ion-optics calculation are shown in Fig. 2. In addition to the characteristic rays, rays representing mass resolving powers of 350, 600 and 900 are shown. Notice the leftright asymmetry in 2 Design of the recoils separator, 3 lon optics owing to the $\mathrm{HO}$ aberrations. The specifications of the magnets and other system components like dimensions, good field regions, etc. are derived from the optimized ion-optics. 


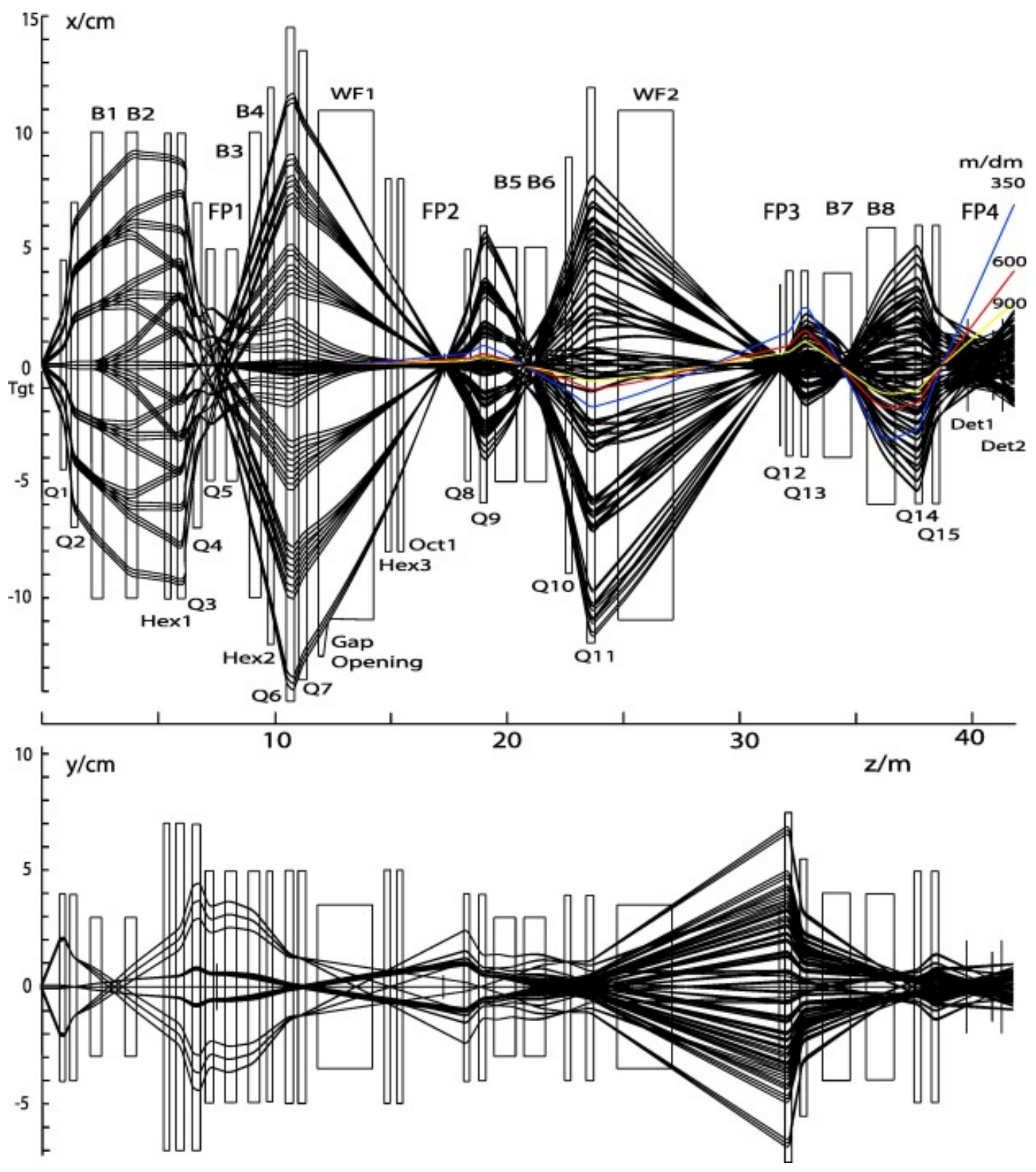

Fig. 2. The ion optics of SECAR for the layout shown in Fig. 1. Shown are 189 characteristic rays in the horizontal and vertical planes in the upper and lower panel, respectively. The horizontal mass resolving power is 508 at FP2 and 767 at FP3.

\section{Project status}

SECAR is designed to allow construction in two phases. Phase 2 is the complete system as described above. In Phase 1 Section 3 with Wien filter WF2 is omitted. In this configuration Section 4 follows immediately Section 2 . This is still a complete recoil separator but with a reduced mass resolving power of $\approx \mathrm{m} / \mathrm{dm}=500$ instead of the design goal of 750 . This would limit the mass range that can be studied to $\approx A<40$. At present SECAR Phase 1 is funded, but we will apply for additional funds to be able to construct the full Phase 2 system. Based on the conceptual design report, requests for quotations with manufacturing specifications have been produced and were sent to potential manufacturers. Quotations have 
been received and a vendor has been selected. SECAR is scheduled to become operational in 2021.

\section{Funding}

This work has been supported by the Dept. of Energy Office for Nuclear Physics, by JINA under grants NSF PHY 08-22648, and NSF PHY-1430152 (JINA Center for the Evolution of the Elements).

This manuscript has been authored in part by UT-Battelle, LLC, under contract DE-AC0500OR22725 with the US Department of Energy (DOE). The US government retains and the publisher, by accepting the article for publication, acknowledges that the US government retains a nonexclusive, paid-up, irrevocable, worldwide license to publish or reproduce the published form of this manuscript, or allow others to do so, for US government purposes. DOE will provide public access to these results of federally sponsored research in accordance with the DOE Public Access Plan (http://energy.gov/downloads/doe-public-access-plan).

\section{References}

1. J. José, M. Hernanz, C. Iliadis. Nucl. Phys. A, 777 (2006), p. 550

2. H. Schatz, K.E. Rehm. Nucl. Phys. A, 777 (2006), p. 601

3. K.A. Chipps, et al. Nucl. Instr. Meth. Phys. Res., A763 (2014), p. 553

4. D. Hutcheon, et al. Nucl. Instr. Meth., A498 (2003), p. 190

5. C.J. Gross, et al. Nucl. Instr. Meth. Phys. Res., A450 (2000), p. 12

6. M.S. Smith, C. Rolfs, C.A. Barnes. Nucl. Instr. Meth. Phys. Res., A306 (1991), p. 233

7. M. Couder, et al. Nucl. Instr. Meth. Phys. Res., A506 (2003), p. 26

8. D. Schurmann, et al. Nucl. Instr. Meth. Phys. Res., A531 (2004), p. 428

9. M. Couder, G.P.A. Berg, et al. Nucl. Instr. Meth. Phys. Res., A587 (2008), p. 35

10. M. Berz, COSY Infinity, <http://www.bt.pa.msu.edu/index files/cosy.htm>. 